A 11102489763

NATL INST OF STANDARDS \& TECH R.I.C.

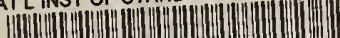

A11102489763

A11 102489763

Nyyssonen, Diana/Linewidth calibration NBS-P

\title{
Linewidth Calibration for Bright-Chromium Photomasks
}

\begin{tabular}{|c|c} 
Reference & NBS \\
PUBLICATIONS
\end{tabular}

D. Nyyssonen

U.S. DEPARTMENT OF COMMERCE

National Bureau of Standards

National Engineering Laboratory

Center for Electronics and Electrical Engineering

Semiconductor Electronics Division

Gaithersburg, Maryland 20899

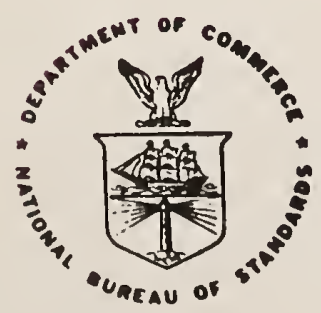

$Q C$

JEPARTMENT OF COMMERCE

100 IAL BUREAU OF STANDARDS

.456

$86-3357$

1986 
NBSIR 86-3357

00

LINEWIDTH CALIBRATION FOR

BRIGHT-CHROMIUM PHOTOMASKS

D. Nyyssonen

U.S. DEPARTMENT OF COMMERCE

National Bureau of Standards

National Engineering Laboratory

Center for Electronics and Electrical Engineering

Semiconductor Electronics Division

Gaithersburg, Maryland 20899

May 1986

U.S. DEPARTMENT OF COMMERCE, Malcolm Baldrige, Secretary NATIONAL BUREAU OF STANDARDS, Ernest Ambler, Director 
Table of Contents

Page

Abstract . . . . . . . . . . . . . . . . . . 1

Introduction . . . . . . . . . . . . . . . . . . 1

AR-Chromium vs. Bright-Chromium . . . . . . . . . . . . 2

The Effect of Variation in Material Parameters . . . . . . . . . . . . . 4

The Effect of Edge-Detection Criterion . . . . . . . . . . . . . . 4

The Effect of Flare . . . . . . . . . . . . . . . . . . . 5

The Effect of Detector Response . . . . . . . . . . . . . . . . . 6

Use of the Bright-Chromium SRM 476 . . . . . . . . . . . . . . 6

Acknowledgments . . . . . . . . . . . . . . . . 7

References . . . . . . . . . . . . . . . . . . 8

List of Figures

1(a). Experimental profile of an AR-chromium line in transmitted light using the

NBS photomask calibration system . . . . . . . . . . . 9

1(b). Experimental profile of a space on a bright chrome photomask . . . . . . 10

1(c). Experimental profile of a line on a bright chrome photomask with flare . . . . 11

1(d). Experimental profile of an AR-chromium line in reflected light . . . . . 12

1(e). Experimental profile of a BC-chromium line in reflected light . . . . . . 13

2. Definition of parameters $T$ (or $R$ ) and $\phi$ used to characterize line objects . . . 14

3. Error $\Delta$ resulting from use of the wrong threshold $T_{E}$ resulting from mismatch of an AR standard to a BC photomask . . . . . . . . . . . 15

4(a). Transmitted light optical parameters $T$ and $\cos \phi$ for different materials . . . 16

4(b). Comparable reflected light optical parameters $R$ and $\cos \phi$ for the same materials 16

5 (a). Plot of the coherent threshold $T_{C}$ as a function of relative transmittance . . 17

$5(\mathrm{~b})$. Same as figure $5(\mathrm{a})$ except for the partially coherent case of matched condenser and objective N.A.'s . . . . . . . . . . . . 18

6. Effect of flare $F$ on the edge detection threshold and the resulting edge detection error when the dc background is not automatically removed . . . . . . . 19

7. Experimental profile of a bright-chromium line profile with flare removed . . . 20 


\author{
D. Nyyssonen* \\ National Bureau of Standards \\ Gaithersburg, MD 20899
}

\begin{abstract}
Linewidth measurement errors are introduced when an anti-reflective (AR) chromium photomask standard such as the NBS SRM 474/475 is used to calibrate an optical linewidth measurement system for subsequent measurements on another material such as bright chromium whose optical properties (index of refraction, thickness, reflectance, and edge geometry) do not match those of the calibration standard. In addition to differences in the optical properties of the materials, the magnitude of these errors varies from system to system and depends upon resolution, choice of edge-detection criterion, flare light in the optical system, and detector response. These errors are greatest when measurements are made in reflected light due to the greater sensitivity to the mismatch in optical parameters of the materials between the calibration standard (AR chromium) and the material to be measured (bright chromium). This report, therefore, recommends use of transmitted light for linewidth measurements on photomasks and as close a match as possible between the material parameters of the calibration standard and those of the part being measured in order to ensure a realistic assessment of the accuracy and precision of subsequent measurements.
\end{abstract}

KEY WORDS: critical dimensions; linewidth measurement; microlithography; microscopy; optical metrology; photomask calibration; scanning microscopy.

\title{
INTRODUCTION
}

The National Bureau of Standards (NBS) has provided to the integrated-circuit (IC) industry for more than five years an anti-reflective (AR) chromium photomask linewidth Standard Reference Material (SRM) [1] with nominal dimensions between 1 and $10 \mu \mathrm{m}$. Because the response of optical linewidth measurement systems is affected by the mate-

* Currently with CD Metrology, Inc., Germantown, MD 20874 
rial properties, measurement errors may be introduced when an AR-chromium standard is used to calibrate a system for subsequent measurements on a different material such as a bright-chromium (BC) photomask or reticle. This report is intended to describe the nature of these errors and discuss how to eliminate or reduce them through proper use of the bright-chromium photomask SRM 476.

Errors in the transfer of linewidth calibrations from one material to another occur because of the optical system response, the edge-detection criterion used, and the difference in optical constants of the materials. Whether used in transmission or reflection, most conventional optical linewidth measurement systems produce an image profile either by slit scanning or from a video camera and apply some fixed criteria such as a $50 \%$ or other threshold for edge-detection and linewidth measurement. Use of a fixed edge-detection threshold, ignoring changes in the edge image structure from one material to another, does not allow the measurement system to compensate for changes in materials and, therefore, results in linewidth measurement errors. These material-dependent errors produce what might be called a "material-dependent micrometer."

The magnitude of these errors varies from system to system and depends on the optical parameters of the system (resolution, aberrations, coherence parameter, detector response, etc.) as well as the materials and edge-detection criteria used. These errors are generally larger for reflected light measurement systems than for transmitted light because of the increased sensitivity of the reflected light image to small changes in the optical parameters of the materials (index of refraction, thickness, etc.). Although this report is directed at bright-chromium photomask calibration, the effects described are present to a greater or smaller degree for see-through chromium and AR-chromium materials that differ in composition or thickness from the NBS AR-chromium SRM.

The best method of reducing or eliminating such errors for a given system is to match the calibration standard (materials, thickness of the patterned material, edge geometry, etc.) as closely as possible to the part to be measured.

AR-Chromium vs. Bright-Chromium

As an illustration of the effects of the difference in AR-chromium and bright-chromium optical properties, figures 1 (a-e) compares edge image profiles from the NBS photomask- 
(transmitted light) and laser (reflected light) calibration systems. These systems have both been previously described in the literature $[2,3]$. The system properties of importance here are the high degree of spatial coherence, diffraction-limited optics, and limited spectral bandwidth.

In transmitted light, both the AR- and bright-chromium photomasks, figures 1 (a) and (b), appear nearly opaque. The only difference is a small amount of flare light (the nonimage forming (extraneous) light reflected by the bright surface of the photomask) which is apparent in the line area of figure 1 (c). Flare adds an incoherent background to the image of opaque lines in a clear surround and appears as a slight loss of contrast. The NBS systems have been designed to minimize the effect of flare light and for AR-chromium measured in transmitted light adds approximately $1 \%$ to the background illumination. Systems encountered in commercial use have been known to add as much as $10 \%$. The effect of flare on the measurement varies with detector response, resolution, and edgedetection criterion used as will be shown.

In reflected light, figures $1(\mathrm{~d})$ and (e), these $\mathrm{AR}$ - and $\mathrm{BC}$-photomask materials vary considerably both in absolute reflectance, $30 \%$ (AR) vs. $65 \%(\mathrm{BC})$, and in the optical parameters of the materials. In figures $1(\mathrm{~d})$ and (e), a distinct difference in the edge image structure can be observed. The optical parameters of importance in determining the image structure, relative reflectance and optical phase difference at the edge, are defined in figure 2.

The effect of a fixed edge-detection criterion is illustrated in the following example in which the effects have been slightly exaggerated to make the discussion clear. Assume that an AR-chromium photomask has been used previously to calibrate the measurement system and that the fixed edge-detection threshold has been adjusted to yield the correct measurements on this AR-chromium "standard." If this same edge-detection threshold is then used on the BC-photomask as shown in figure 3, an error "delta" occurs because the true edge location does not correspond to this threshold because of the change in image structure due to the change in optical properties from $A R$ to $B C$.

As already noted, the magnitude of "delta" depends on the material parameters T (or R) and $\phi$, the resolution of the imaging system (edge image slope), the edge-detection criteria used, and the linearity of the detector response. Results of a study to assess the magnitude 
of these effects are given in reference 4.

The Effect of Variation in Material Parameters

Changes in the optical parameters of the photomask material, relative transmittance (or reflectance), and phase difference as shown in figure 2 cause the observed edge image profile or waveform to change. The relation between a given threshold or feature and the true edge location also changes. The change increases with increasing differences in the $\mathrm{T}$ (or $R$ ) and $\phi$ parameters. (See for example, figs. 4(a) and (b).) Note that the differences in optical parameters for these materials is greater in reflection.

These differences translate into a required change in the edge-detection threshold used in order to maintain accurate measurements. A discussion of the variation in the edgedetection threshold is given in reference 5 and results are reproduced in figure 5 for different coherence parameters. The magnitude of the actual dimensional measurement error introduced, however, is a function of the resolution of the measurement system and the particular edge-detection algorithm used.

\section{The Effect of Edge-Detection Criterion}

An extended discussion of the combined effect of material parameters and edge-detection criterion may be found in reference 5 for wafer measurements where the variations found in material parameters are much larger than in thin-layer photomasks. Here, we use the example given in figure 3 and compare the errors associated with use of the different edge-detection criteria listed in Table 1.

In Case I, the AR-chromium photomask SRM is used to select the edge-detection threshold which is then incorrectly used for measurement of the BC-photomask. The resulting measurement errors are shown in Table I as a function of the objective numerical aperture (N.A.) and coherence parameter (S)* In Case II, the $50 \%$ threshold is used arbitrarily for all measurements, and an improper correction for the bright-chromium photomask is made based on a calibration to the AR standard. The measurement errors resulting from this case are also shown in Table I. The calculations leading to the values in Table I assume diffraction-limited optics. With aberrations present, an approximate value of the error can

* Ratio of numerical aperture of condenser lens to objective lens. 
Table I. Theoretically calculated linewidth measurement errors (in $\mu \mathrm{m}$ ) for given N.A. of the objective lens and coherence parameter S; reflected light, diffraction limited optics, and ideal detector assumed. Errors are shown for a space. For lines, the errors are of the same magnitude, but opposite sign. In real systems, errors tend to be larger and may not be of the same magnitude for both lines and spaces.

\begin{tabular}{|l|l|l|l|l|}
\hline \multicolumn{2}{|c|}{ N.A. $=0.90$} & \multicolumn{2}{c|}{ N.A. $=0.60$} \\
\hline & $\mathrm{S}=0.66$ & $\mathrm{~S}=1.0$ & $\mathrm{~S}=0.66$ & $\mathrm{~S}=1.0$ \\
\hline $\begin{array}{l}\text { Case I - } \\
T_{E} \text { set by the } \\
\text { calibration } \\
\text { standard. }\end{array}$ & +0.06 & +0.04 & +0.09 & +0.05 \\
\hline $\begin{array}{l}\text { Case II - } \\
T_{E}=50 \%\end{array}$ & +0.04 & +0.04 & -0.16 & +0.05 \\
\hline
\end{tabular}

be estimated by looking at values for a lower resolution system, i.e., a lower N.A.

The Effect of Flare

As already indicated, flare (the term is used here to describe extraneous light reflected by the bright surface of the photomask) adds an incoherent background to the image, thus shifting the image intensity with respect to the detector response curve. Video systems which automatically subtract out a dc background in order to improve the contrast or visibility of the image may show little or no error due to flare. There may still be an error due to the mismatch in optical parameters in this case.

When the dc-background is not subtracted out, flare will introduce a change in the threshold corresponding to edge location as illustrated in figure 6 . In this case, no significant amount of flare is present when the AR standard is used for calibration, but is present for bright-chromium lines in a clear surround, and this causes the wrong edge-detection threshold to be used. The resulting linewidth error depends upon the amount of flare and the resolution or image edge slope. In the NBS photomask calibration system, $1 \%$ flare introduces an error of less than $0.01 \mu \mathrm{m}$. Increased amounts of flare, as much as $10 \%$ or more, in a lower resolution system can cause errors as large as tenths of micrometers. 
In transmitted light, the $\mathrm{AR}$ - and $\mathrm{BC}$-chromium photomasks are so closely matched in absolute transmittance that no effect due to detector response is seen when substituting one for the other. However, in a reflected light system, the enormous difference in absolute reflectivity of the materials requires an adjustment of the illumination level by a multiplicative factor of more than 2 in order to maintain the image intensity on the same portion of the detector response curve (no change in gain or sensitivity).

Therefore, if the detector has sufficient sensitivity to yield a good image of the ARphotomask in reflected light, a neutral density filter with $50 \%$ transmission (0.3 N.D.) could be used and trimmed with a slight change in lamp intensity when measuring the BC-photomask in order to maintain the image intensity on the same portion of the detector response curve. If properly adjusted, any possible error due to detector response would be minimized. What more frequently happens, however, is that a change in gain or sensitivity adjustment is made to compensate for the change in reflectivity. If the detector response is not a linear function of its input, or the compensation for the difference in reflectance is not made exactly, calibration may be lost and large errors may result. This problem occurs most often in video systems where a factor of 2 change in illumination or image intensity level cannot be tolerated due to the nonlinear detector response and limited response range.

As in the case of flare, a small shift of the image intensity (especially that corresponding to edge location) due to inaccurate compensation for change in reflectivity of the photomask with respect to the detector response curve may or may not produce an error depending upon whether or not the dc background is removed.

It should be apparent from this discussion that the magnitude of these effects are highly dependent on the mode of operation of the measurement system as well as the optical parameters of the photomasks. Again, the problems are minimized (if not wholly eliminated) when transmitted light is used for linewidth measurement on photomasks!

\section{Use of the Bright-Chromium SRM 476}

In order to assist in improving the accuracy and reproducibility of linewidth measurements made on bright-chromium photomasks, NBS is providing SRM 476. It is certified to a 
precision and accuracy comparable to that of SRM 474/475 primarily because of the close match in optical parameters when measurements are made in transmitted light and the small amount of flare light introduced is taken into account by a correction to the edgedetection threshold.

This correction is made by first determining the amount of flare from a comparison of the images of a clear space in an opaque surround and an opaque line in a clear surround such as shown in figures 1 (b) and (c). (The widths of the lines used for this comparison must be at least a few micrometers in order for diffraction effects to be excluded.) In the NBS photomask calibration system, the correction to the coherent edge-detection threshold [2] takes the form:

$$
T_{c}^{\prime}=\frac{I_{M}}{4}\left[1+\left(\frac{I_{O}}{I_{M}}-F\right)+2 \sqrt{\left(\frac{I_{O}}{I_{M}}-F\right)} \cos \phi\right]+F
$$

where $F$ is the amount of flare light normalized to $I_{M}$.

This correction is equivalent to subtracting out the flare light before applying the coherent edge-detection threshold. This technique is demonstrated in figure 7 where, as a result of subtraction of the flare light, the edge image profile for an opaque line becomes identical to that of the clear space shown in figure $1(\mathrm{~b})$.

In transmitted light optical systems, the procedures for the use of SRM 476 are identical to those recommended for the AR-chromium SRM 474/475. In today's automated systems, the measurement system adjustments described in the ASTM Standard Practice for Preparing an Optical Microscope for Dimensional Measurements may not be possible, and the user may have no way of knowing whether his system is optimally set up. In such a case, matching of the material parameters of the standard to the part being measured and generation of a reliable calibration curve based on statistical analysis of repeated measurements are essential to realistic assessment of the accuracy and precision of subsequent measurements.

\section{Acknowledgments}

The author wishes to thank Marilyn J. Dodge for her assistance in compiling the experimental and theoretical profiles used to produce the tables and figures in this report. 


\section{References}

1. NBS SRM 474/5, available from the Office of Standard Reference Materials, Room B311, Chemistry Building, Gaithersburg, MD 20899.

2. D. Nyyssonen, "Theory of Optical Edge Detection and Imaging of Thick Layers," J. Opt. Soc. Am 72, 1425-1436 (1982).

3. D. Nyyssonen, "Calibration of Optical Systems for Linewidth Measurements on Wafers," Opt. Eng. 21, 882-887 (1982).

4. J. M. Jerke and C. E. Wendell, "Use of the NBS AR-Chromium Optical Linewidth Standard for Measurements on Other Types of Chromium Photomasks," Proc. SPIE Vol. 342, 15-26 (1982).

5. D. Nyyssonen, "A Practical Method for Edge Detection and Focusing for Linewidth Measurements on Wafers," Proc. SPIE Vol. 538, 172-178 (1985). 


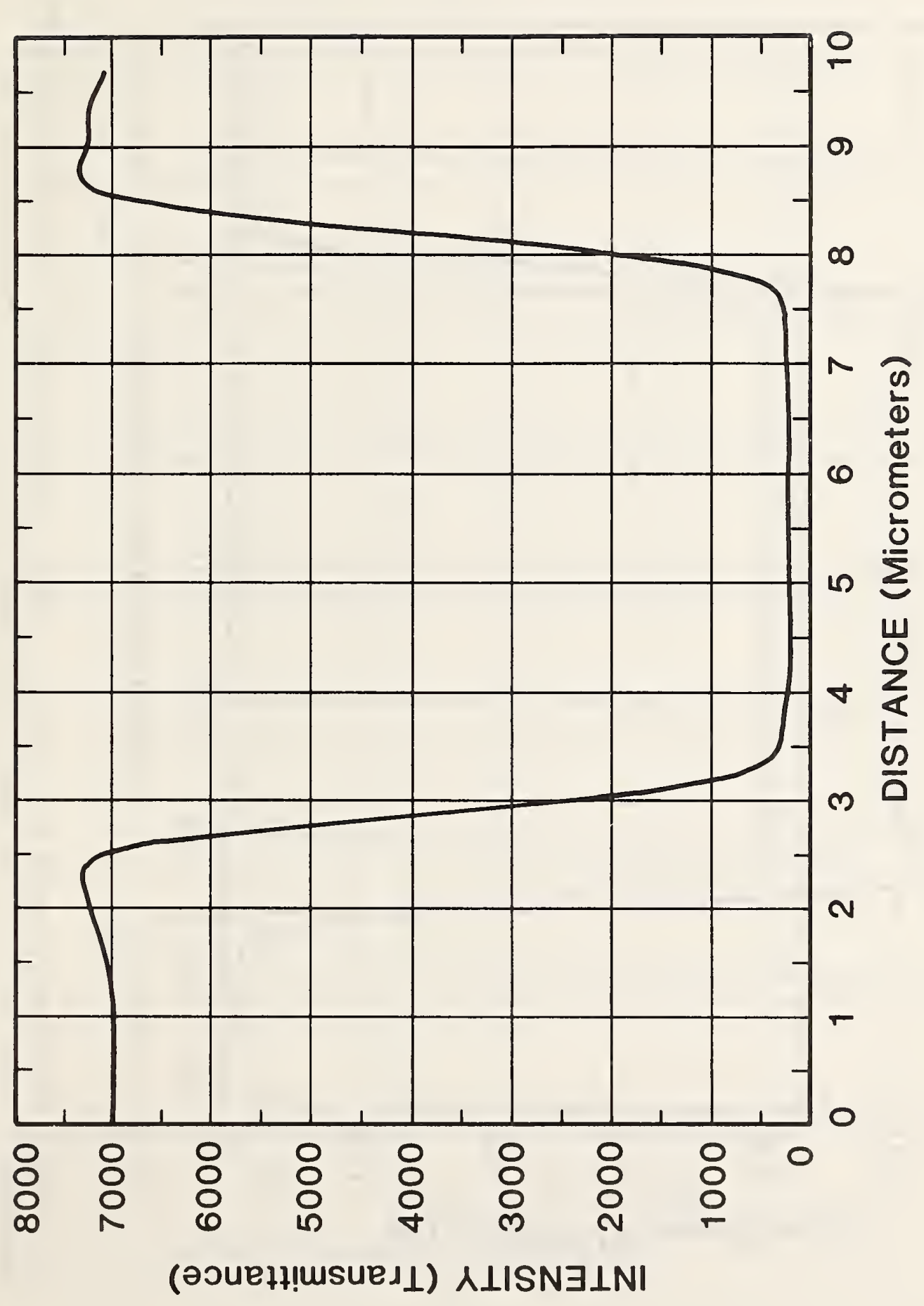

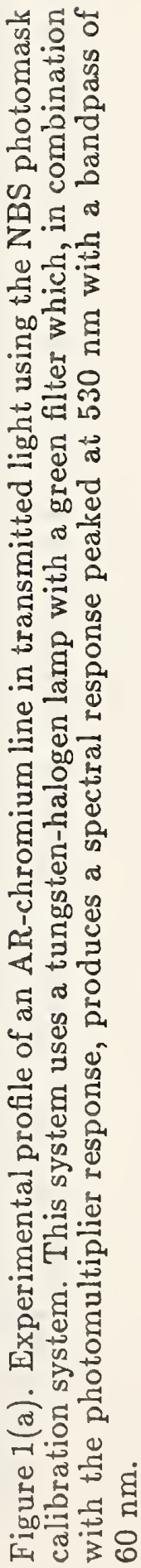




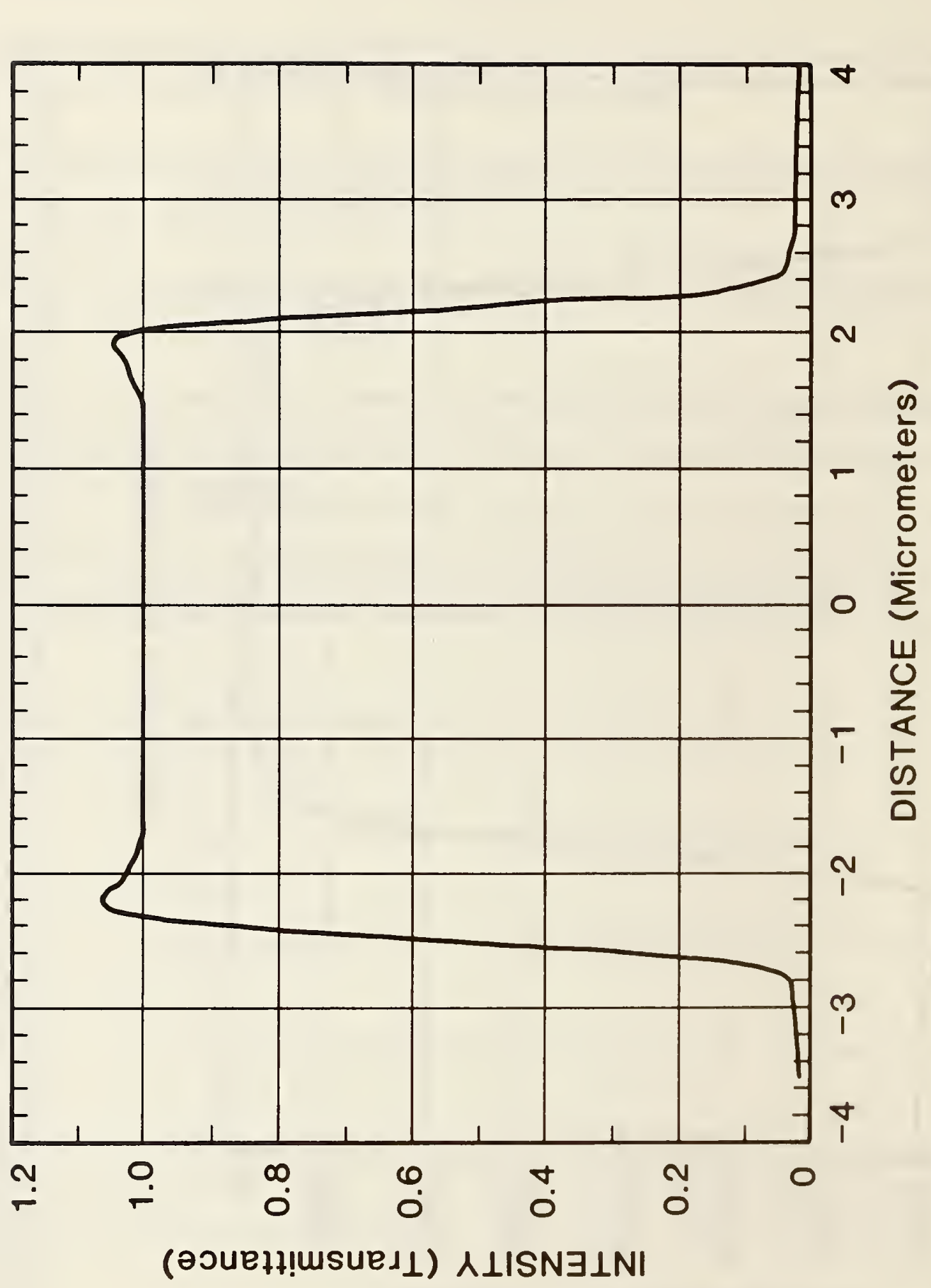






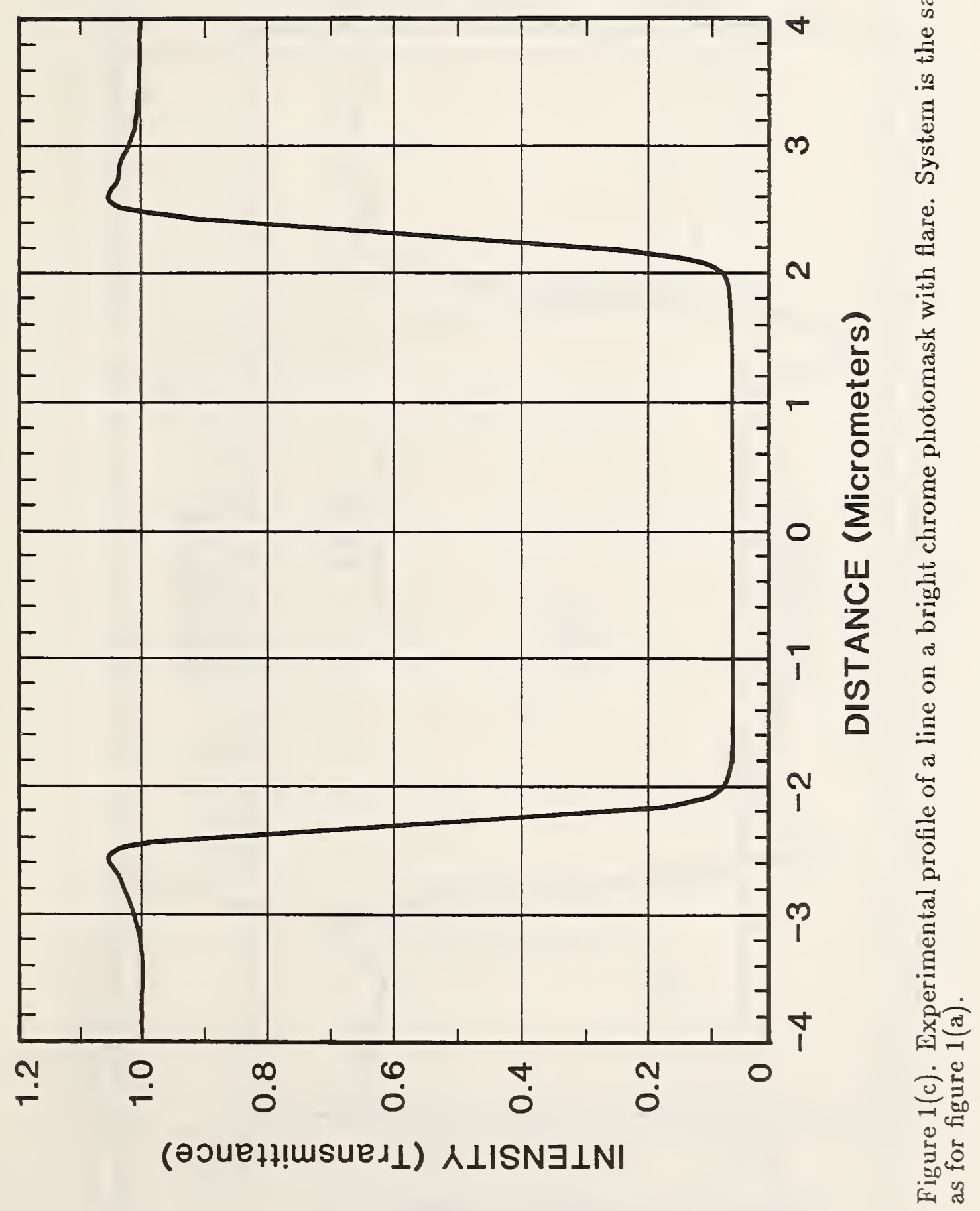






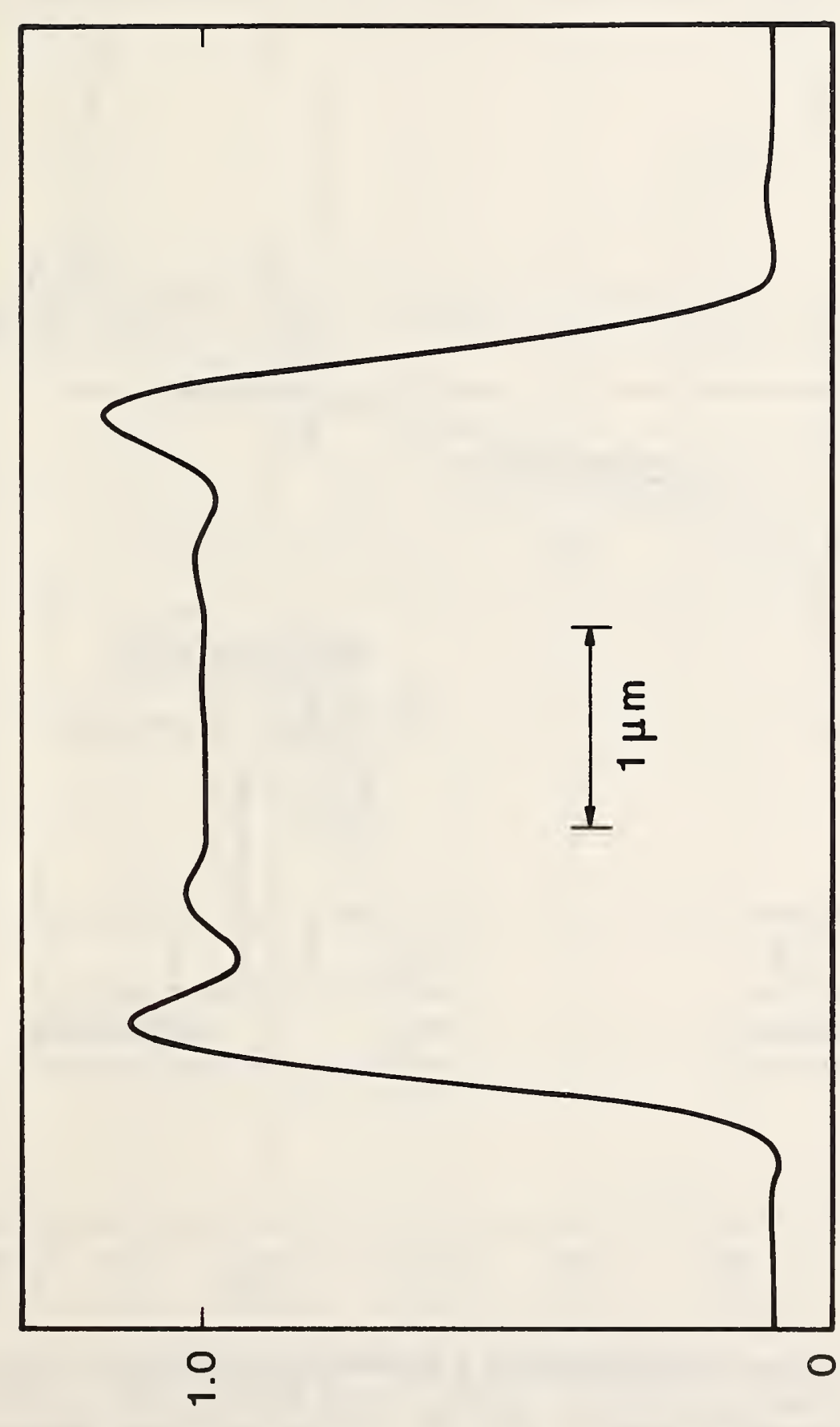

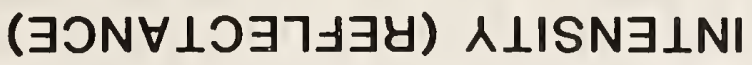

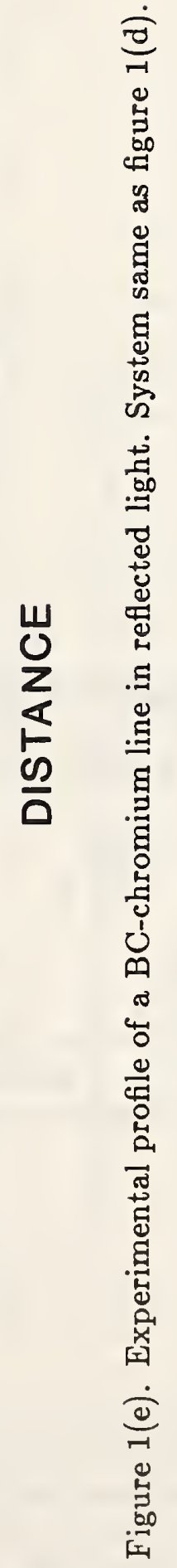






a.

\section{TRANSMITTED}

$\Phi=\phi_{2}-\phi_{1}+k d$

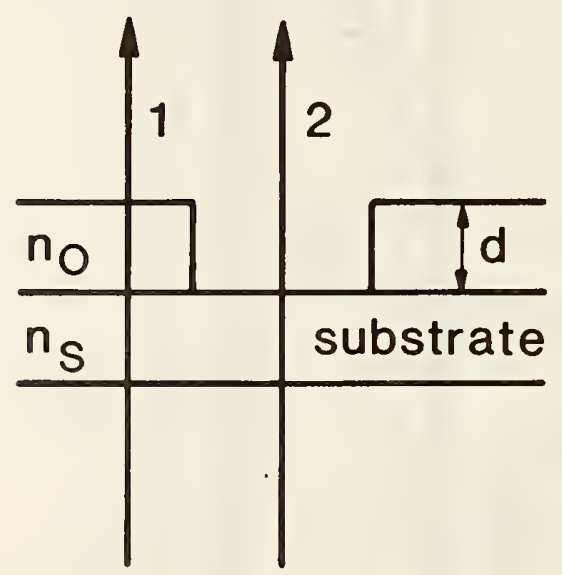

b.
REFLECTED

$\phi=\phi_{2}-\phi_{1}+2 k d$

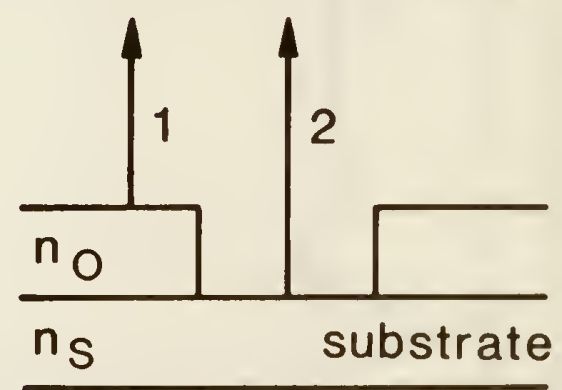

Figure 2. Definition of parameters $T$ (or $R$ ) and $\phi$ used to characterize line objects. (a) Image profile showing $I_{M}$ and $I_{O}$, where $T=I_{O} / I_{M}$. When $T_{E}\left(T_{E}=T_{C} \times I_{M}\right)$ is used for edge detection, the linewidth is given by $X_{2}-X_{1}$. The optical phase difference $\phi$ is determined from the optical path difference between rays 1 and 2 for (b) transmitted light and (c) reflected light. In each case, the values of $\phi_{1}$ and $\phi_{2}$ can be calculated from the given complex indices of refraction $\hat{n}_{o}$ and $\hat{n}_{B}$ and the thickness $d$ of the patterned layer by using the Fresnel equations. 


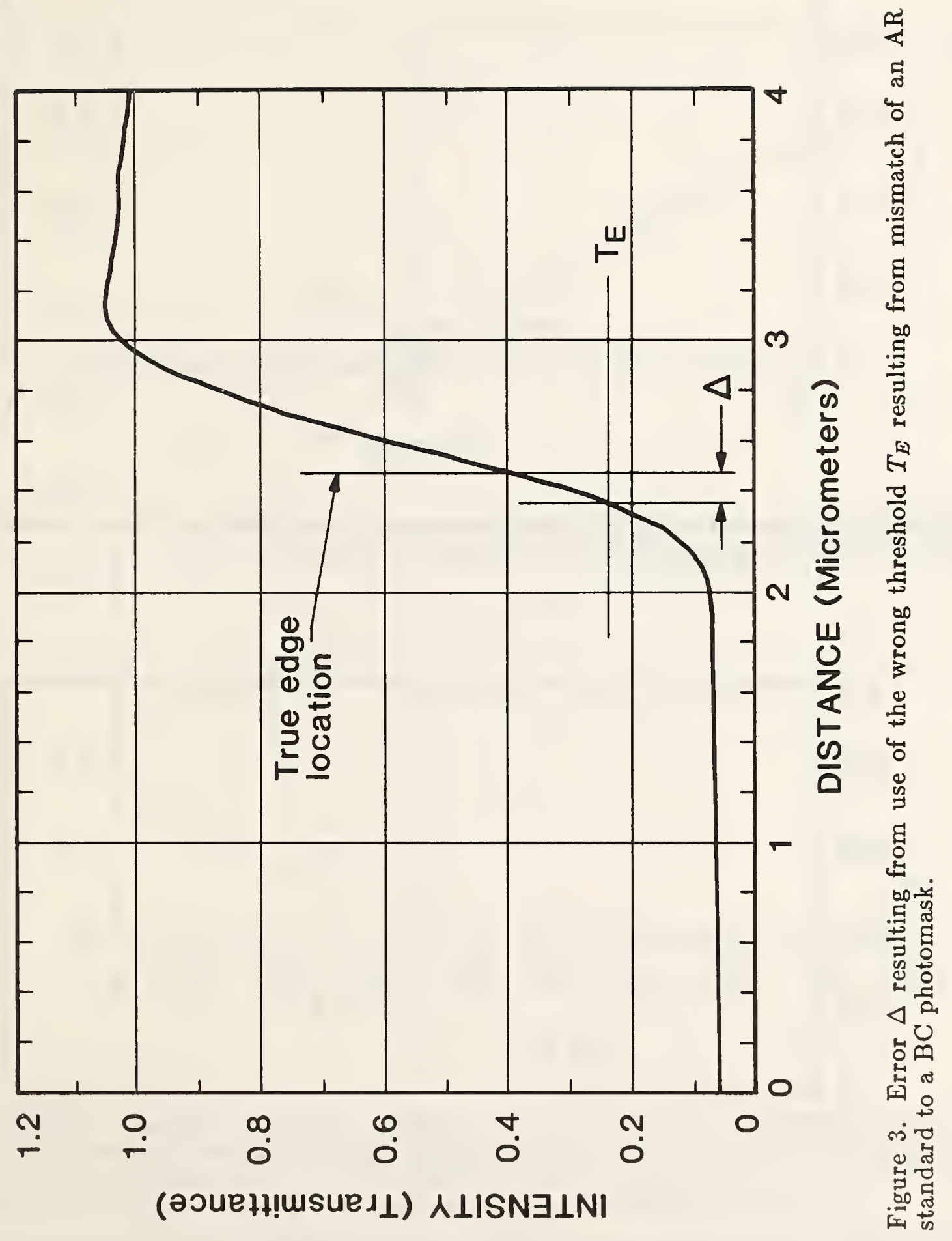




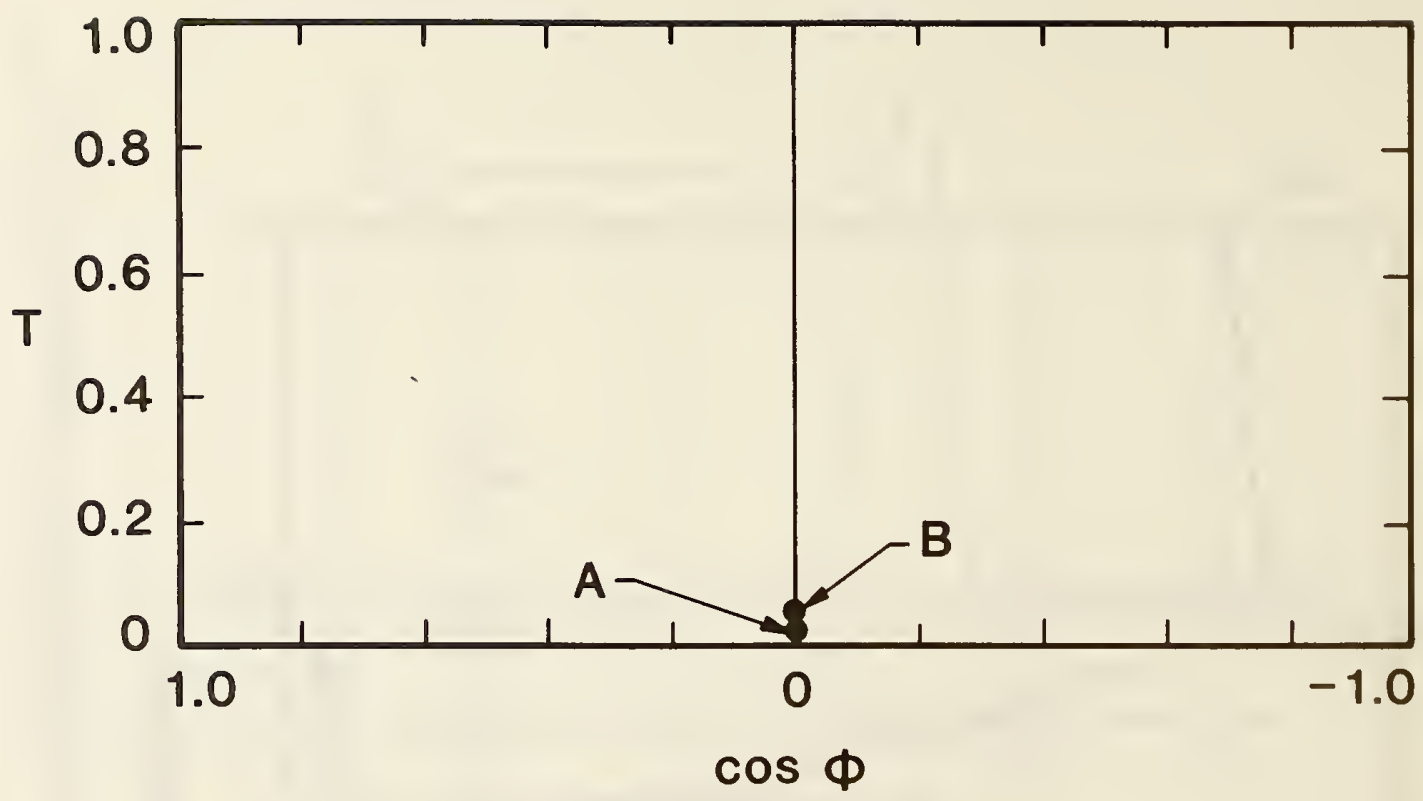

Figure 4(a). Transmitted light optical parameters $T$ and $\cos \phi$ for different materials; A, SRM 474 (AR chromium); B, SRM 476 (bright chromium).

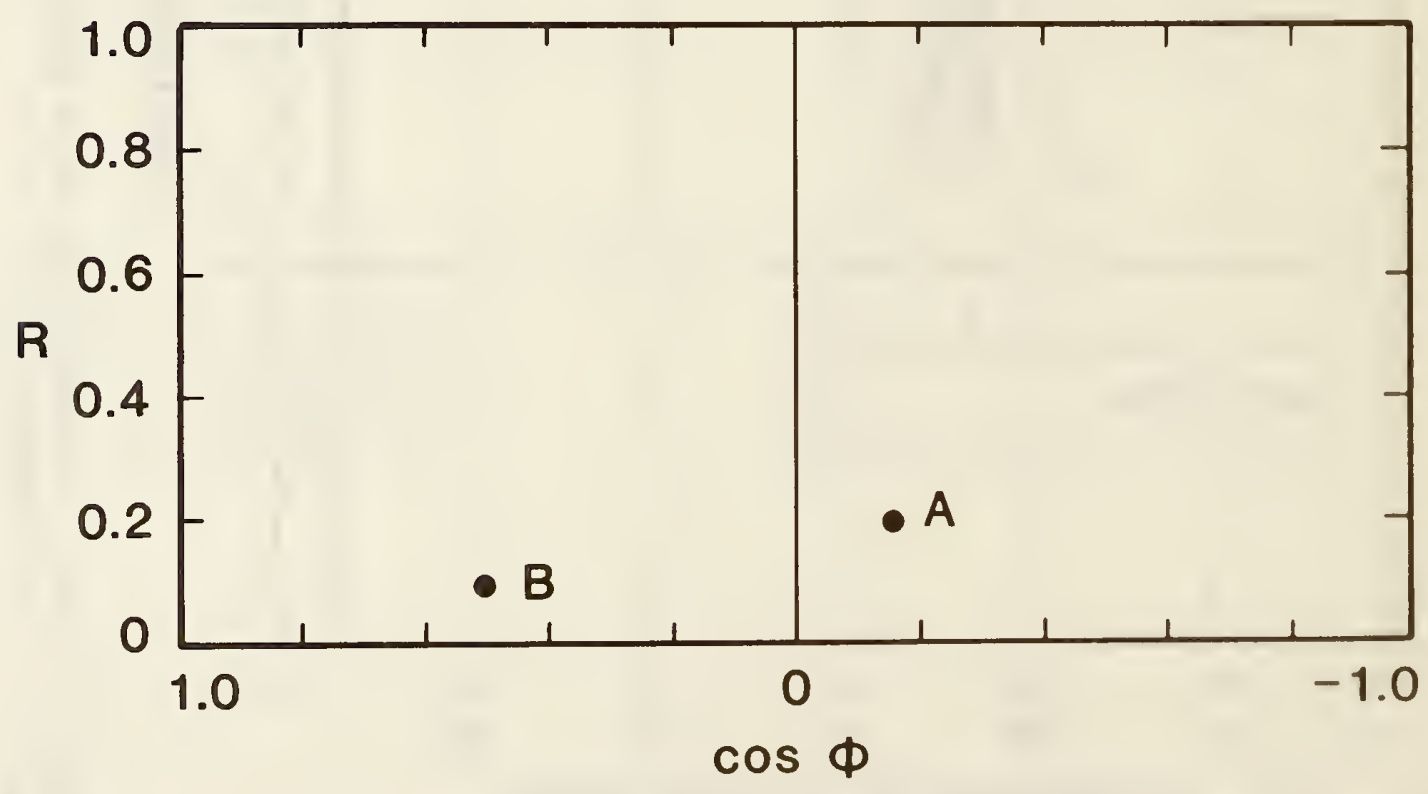

Figure 4(b). Comparable reflected light optical parameters $R$ and $\cos \phi$ for the same materials. 


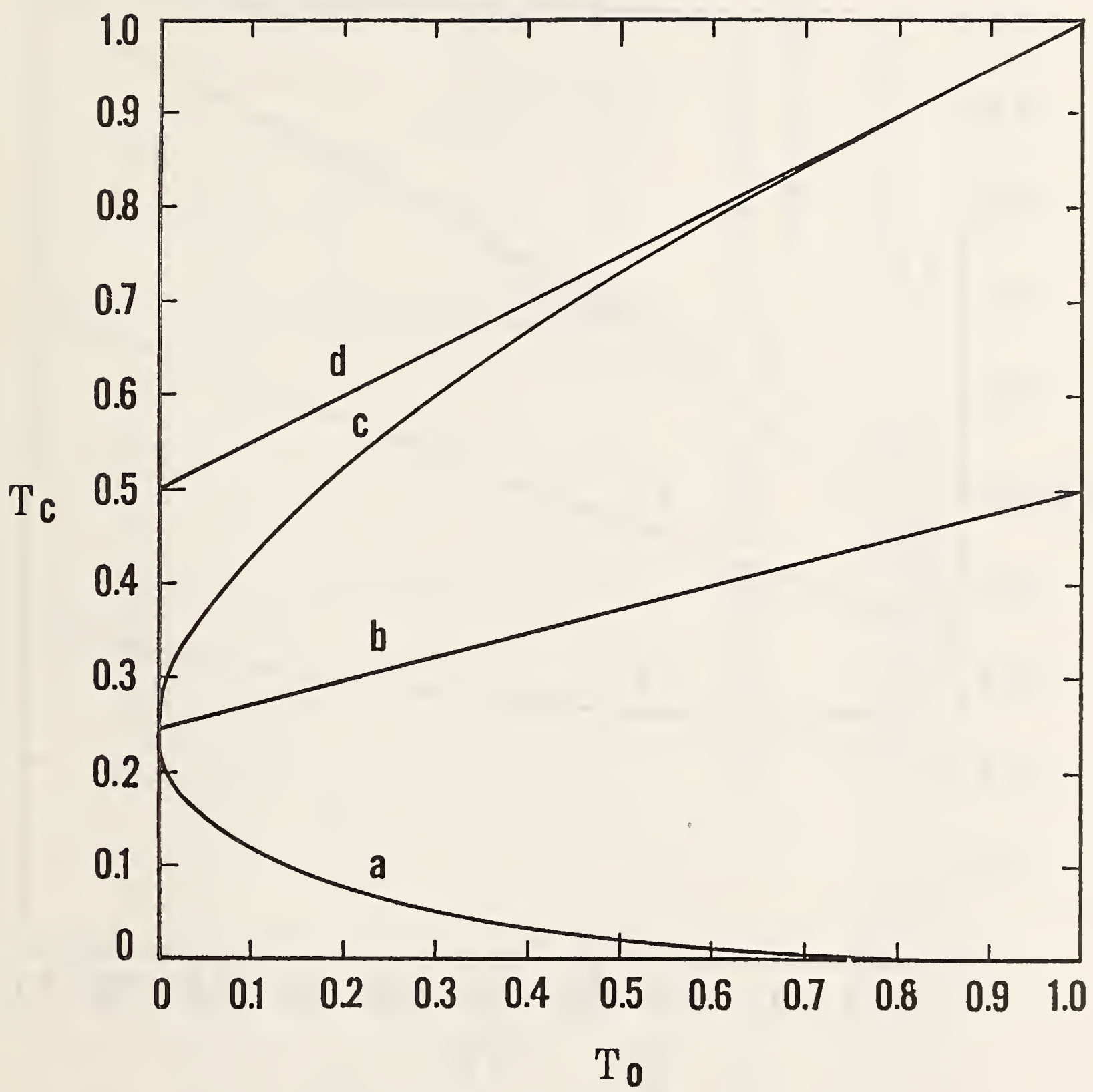

Figure 5(a). Plot of the coherent (objective N.A. $\gg$ condenser N.A.) threshold $T_{C}$ as a function of relative transmittance (or reflectance) $T$ (or $R$ ) for a, $\phi=\pi ; b, \phi=\pi / 2$; and $\mathrm{c}, \phi=0$. The threshold corresponding to incoherent illumination (condenser N.A. $\gg$ objective N.A.) that is insensitive to $\phi$ is shown for comparison (curve d). 


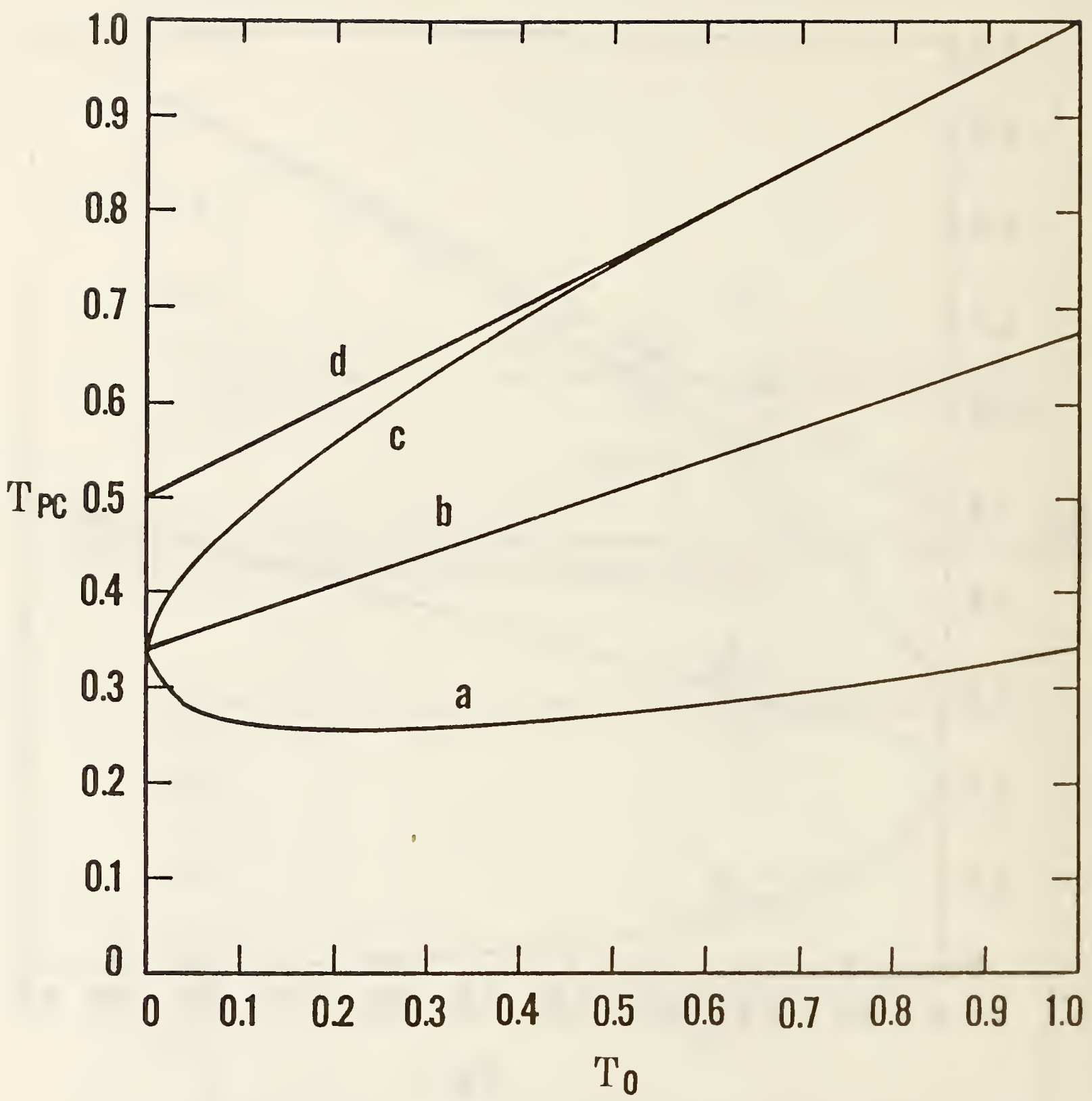

Figure 5(b). Same as figure 5(a) except for the partially coherent case of matched condenser and objective N.A.'s. 


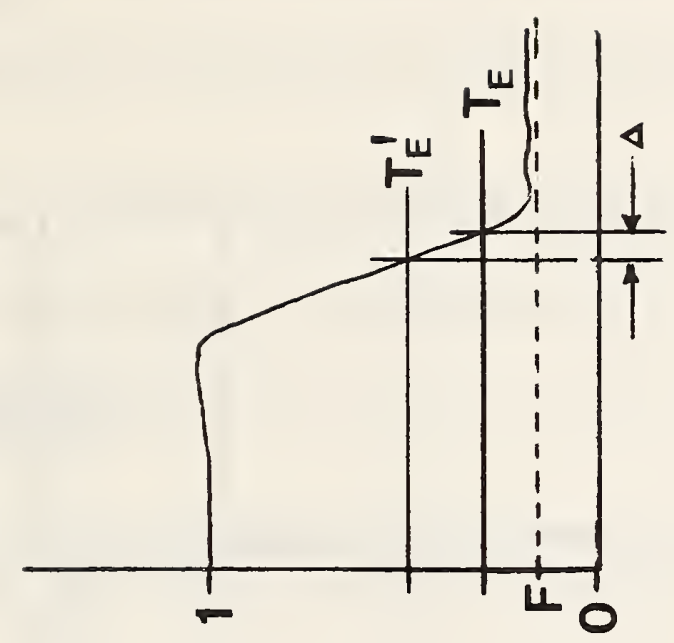



이용



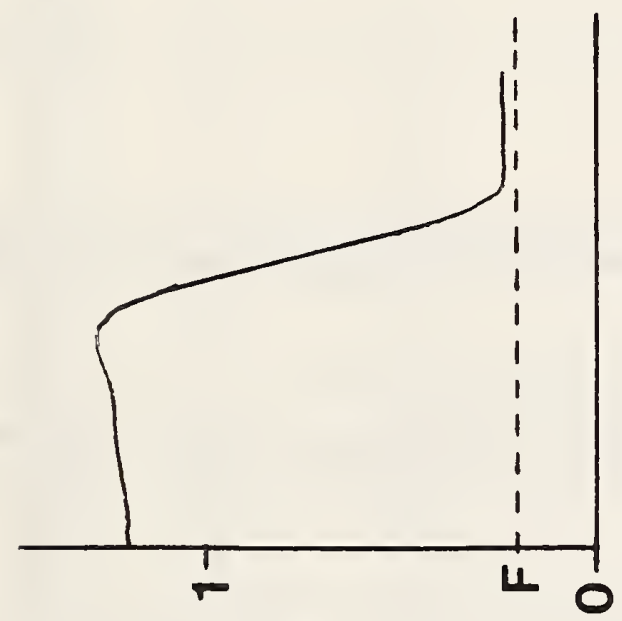

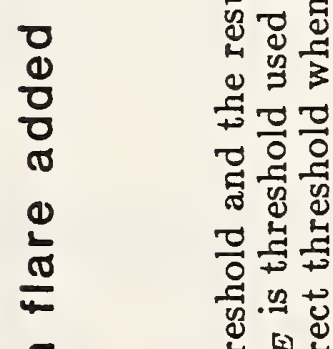



责

i

宅.

娄范

5ㅇํㄱ. 곡

近

윰

蛋.

뭉.

艺言.

造茄实

○.

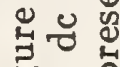

.000

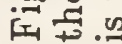

$\wedge \perp I S N \exists \perp N I \exists \wedge I \perp \forall 7 \exists y$ 


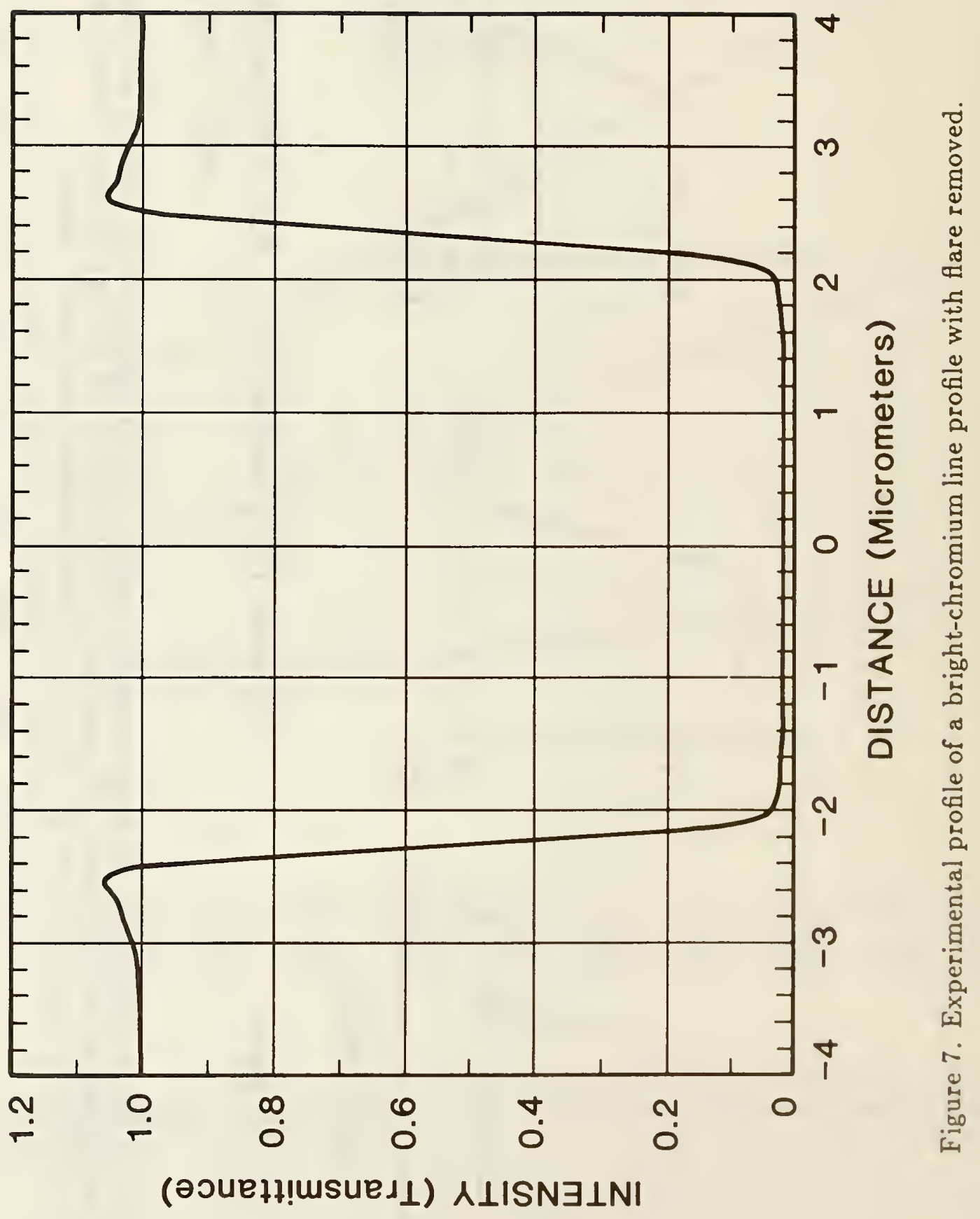




\begin{tabular}{|c|c|c|c|}
\hline $\begin{array}{l}\text { U.S. DEPT. OF COMM. } \\
\text { BIBLIOGRAPHIC DATA } \\
\text { SHEET (See instructions) }\end{array}$ & $\begin{array}{l}\text { 1. PUBLICATION OR } \\
\text { REPORT NO. } \\
\text { NBSIR }-86 / 3357\end{array}$ & 2. Performing Organ. Report Nof & $\begin{array}{l}\text { 3. Publication Date } \\
\text { May } 1986\end{array}$ \\
\hline \multicolumn{4}{|l|}{ 4. TITLE AND SUBTITLE } \\
\hline \multicolumn{4}{|l|}{ 5. AUTHOR(S) } \\
\hline \multicolumn{3}{|c|}{$\begin{array}{l}\text { 6. PERFORMING ORGANIZATION (If joint or other than NBS, see instructions) } \\
\text { NATIONAL BUREAU OF STANDARDS } \\
\text { DEPARTMENT OF COMMERCE } \\
\text { WASHINGTON, D.C. } 20234\end{array}$} & 8. Type of Report \& Period Covered \\
\hline
\end{tabular}

9. SPONSORING ORGANIZATION NAME AND COMPLETE ADDRESS (Street, City, State, ZIP)

10. SUPPLEMENTARY NOTES

[Document describes a computer program; SF-185, FIPS Software Summary, is attached.

11. ABSTRACT (A 200-word or less factual summary of most significant information. If document includes a significant bibliography or literature survey, mention it here)

Linewidth measurement errors are introduced when an anti-reflective (AR) chromium photomask standard such as the NBS SRM $474 / 475$ is used to calibrate an optical linewidth measurement system for subsequent measurements on another material such as bright chromium whose optical properties (index of refraction, thickness, reflectance, and edge geometry) do not match those of the calibration standard. In addition to differences in the optical properties of the materials, the magnitude of these errors varies from system to system and depends upon resolution, choice of edge-detection criterion, flare light in the optical system, and detector response. These errors are greatest when measurements are made in reflected light due to the greater sensitivity to the mismatch in optical parameters of the materials between the calibration standard (AR-chromium) and the material to be measured (bright chromium). This report, therefore, recommends use of transmitted light for linewidth measurements on photomasks and as close a match as possible between the material parameters of the calibration standard and those of the part being measured in order to ensure a realistic assessment of the accuracy and precision of subsequent measurements.

12. KEY WORDS (Six to twelve entries; alphabetical order: copitalize only proper names; and separate key words by semicolons) critical dimensions; linewidth measurement; microlithography; microscopy; optical metrology; photomask calibration; scanning microscopy

13. AVAILABILITY

XX] Unlimited

$\square$ For Official Distribution. Do Not Release to NTIS

[ Order From Superintendent of Documents, U.S. Government Printing Office, Washington, D.C. 20402.

[ Order From National Technical Information Service (NTIS), Springfield, VA. 2216I
14. NO. OF

PRINTED PAGES

$$
24
$$

15. Price $\$ 9.95$ 


